\title{
Port Wine Stain
}

National Cancer Institute

\section{Source}

National Cancer Institute. Port Wine Stain. NCI Thesaurus. Code C3840.

A congenital vascular malformation in the skin (birthmark) characterized by the presence

of dilated capillaries. The affected area of the skin is flat and reddish-purplish in color. 